\title{
ANÁLISIS DE LAS DIMENSIONES DEL PROCESO DE TRANSFERENCIA TECNOLÓGICA COMO MÉTRICA PARA CUANTIFICAR SU ABSORCIÓN EN ENTORNOS ORGANIZACIONALES
}

\section{ANALYSIS OF THE DIMENSIONS OF THE TECHNOLOGY TRANSFER PROCESS AS A METRIC TO QUANTIFY ITS ABSORPTION IN ORGANIZATIONAL ENVIRONMENTS}

\section{Dr. Byron Medina-Delgado*, MS.c Oriana Alexandra López-Bustamante* Dr. Wlamyr Palacios-Alvarado*}

* Universidad Francisco de Paula Santander, Facultad de Ingeniería, Grupo de Investigación y Desarrollo en Electrónica y Telecomunicaciones (GIDET) - Grupo de Investigación en Tecnología, Innovación y Sociedad (GITIS) - Grupo de Investigación en Productividad y Competitividad (GIPyC).

Av. Gran Colombia 12E-96 Colsag, San José de Cúcuta, Norte de Santander, Colombia. $(+57) 75776655$ Ext 202

E-mail: \{byronmedina, orianaalexandralb, wlamyrpalacios $\} @$ ufps.edu.co.

Resumen: La transferencia tecnológica germina como un elemento diferenciador entre las organizaciones, como ventaja competitiva, con el propósito de identificar la tecnología que puede contribuir a la organización favorablemente en su entorno dinámico, a partir de conocimientos tecnocientíficos, para implementar un desarrollo industrializado. Para ello, se empleó un muestreo simple aleatorio de una población determinada, se calcularon medidas de tendencia central y dispersión, a la vez se diseñó un instrumento de investigación que fue validado, a través de pruebas no paramétricas y se estableció su confiabilidad, este documento se diseñó a partir de la sistematización de variables. Los resultados indicaron que en cuanto a transferencia tecnológica, las empresas reconocen la tecnología de manera regular, lo que implica una concepción regular del ciclo de vida de la tecnología, de las fuentes para identificar la tecnología, y una concepción buena del mercado de la tecnología.

Palabras clave: transferencia tecnológica, identificación tecnológica, adquisición tecnológica, asimilación tecnológica, pymes.

\begin{abstract}
The technology transfer germinates as a differentiating element between organizations, as a competitive advantage, with the purpose of identifying the technology that can contribute to the organization favorably in its dynamic environment, from techno-scientific knowledge, to implement an industrialized development. For this purpose, a simple random sampling of a determined population was used, central tendency and dispersion measures were calculated, at the same time a research instrument was designed that was validated, through non-parametric tests and its reliability was established. This document was designed based on the systematization of variables. The results indicated that in terms of technology transfer, the companies recognize the technology on a regular basis, which implies a regular conception of the technology life cycle, of the sources to identify the technology, and a good conception of the technology market.
\end{abstract}

Keywords: Technology transfer, technology identification, technology acquisition, technology assimilation, SMEs. 


\section{INTRODUCCIÓN}

Las pymes (pequeñas y medianas empresas), tanto en América Latina como en Europa, son consideradas como agentes económicos de cambio, pues contribuyen significativamente con la generación de empleo y un aporte al PIB (Producto Interno Bruto) de la región (CEPAL, 2011). Siendo así, para generar una contextualización de la situación actual de las pymes en Colombia, se tiene que, en el tejido empresarial colombiano las pymes representan el $96.4 \%$ de los establecimientos, un aproximado del $63 \%$ del empleo a nivel nacional y el $45 \%$ de la producción manufacturera, el $40 \%$ de los salarios y el $37 \%$ del valor agregado, esto es más de 650000 empresarios cotizando seguridad social (F.J. Vélez V, 2006).

Sin embargo, según (CEPAL, 2011) en Latinoamérica y el Caribe, las empresas de tamaño mediano, presentan un escaso dinamismo y, atraso tecnológico, lo que evidencia una baja participación en exportaciones y una marcada brecha de productividad, respecto a las grandes empresas. Por otra parte, se ha logrado identificar que la innovación, apoyada en los avances científicos tecnológicos, involucrados a la producción, desarrolla o mejora los procesos y productos existentes. La modernización tecnológica, promueve el crecimiento de la productividad y la competitividad de las empresas, características fundamentales en un mundo globalizado de permanente cambio, reformulando la dinámica de los negocios (Rodríguez, 2014). En América Latina, son escasas las empresas medianas, que cuentan con una estrategia de desarrollo y consolidación, fundamentada en la transferencia tecnológica, y las empresas que la tienen, normalmente responden a mecanismos informales, no al resultado de una rigurosa planificación (OCDE/CEPAL, 2012).

Avanzar hacia un escenario de crecimiento empresarial sostenible con mayor igualdad, supone construir mecanismos que permitan densificar la matriz productiva, diversificarla en términos de actividades de alta productividad e incorporar en ellas conocimiento (Del Río et al., 2016). La fuerza motriz de esa transformación, es la generación de conocimiento, promoción de innovaciones y nuevas tecnologías, a lo largo del sistema productivo. En el corazón de ese proceso, se encuentran las Tecnologías de la Información y las Comunicaciones (TIC) y la difusión del paradigma digital. Esta disyuntiva, es fundamental para definir las políticas tecnológicas y de sociedad de la información (CEPAL, 2013).

La base para la innovación tecnológica, es estar a la vanguardia en los procesos y productos, la mejor manera, es que las organizaciones estén atentas a los cambios y la evolución del desarrollo tecnológico, a través de un proceso de vigilancia tecnológica (Jaimes et al., 2011).

El desafío más importante al cual se están enfrentando los empresarios, es alcanzar mayores niveles de competitividad en un entorno cada vez más globalizado, enmarcado por los tratados comerciales, donde, las demandas de tecnologías de la información, electrónica y comunicaciones, automatización y, robótica, así como, el desarrollo de materiales, diseño de procesos industriales, y filosofías de producción, marcan el progreso de una nueva cultura y etapa industrial (Colciencias, 2005). Entre otras, esto es el efecto látigo en términos de tecnología derivados por los avances e implantación de nuevos sistemas productivos a partir de la industria 4.0 (León, J. A. P, 2015; Roncancio-Lozano, M. A. et al, 2015)

Es por ello y de acuerdo a las tendencias internacionales, que el Ministerio de Ciencias desarrolló una propuesta de política nacional colombiana de ciencia, tecnología e innovación (CONPES, 2015), la cual considera que el desarrollo de actividades de ciencia, tecnología e innovación, requiere de personas altamente calificadas que pueden incrementar la productividad, así como, generar mayor producción de conocimiento y desarrollo tecnológico. Reconoce que la función que cumple la investigación, es expandir la frontera del conocimiento científico tecnológico, para dar respuesta a las necesidades, oportunidades sociales y de desarrollo productivo del país. Esta misma política nacional de ciencia y tecnología, define el aparato productivo, como el principal agente innovador, planteando como objetivo, generar condiciones para la cooperación de los sectores productivo, público y, privado, por medio de la transferencia de conocimiento y tecnología (TCT) (Morales-Castro, J. A., 2015; Becerra, E. S., 2017)

De acuerdo con lo anterior, esta investigación pretende empoderar a la mediana empresa de la ciudad de Cúcuta, para que promueva la transferencia tecnológica, en donde, según (Kim, 
1997; Ulrich, 2000) es el empresario, uno de los actores más beneficiados.

El desarrollo tecnológico de la mediana empresa de Cúcuta, no solo beneficia a los empresarios, también favorece al recurso humano, que se apropie de los conceptos expuestos en esta investigación; porque las empresas que implementen la transferencia tecnológica, requieren de personal competente, que recibe mayor remuneración económica por su trabajo. Por lo tanto, el impacto social de esta propuesta, incluye a los empresarios y trabajadores de la mediana empresa, que incorporen como estrategia competitiva la transferencia tecnológica (Rojas, P. R. S, 2016; Rodríguez, L. J., y Gamboa-Suárez, R., 2018; Moreno, J. R. V., et al., 2016).

Finalmente, el presente trabajo investigativo tiene por objeto describir e identificar los elementos de transferencia tecnológica en la mediana empresa de la ciudad de Cúcuta, empleando para efectos metodológicos un instrumento validado por expertos, el cual se aplicó a una muestra representativa. La percepción de los directos en cuanto a transferencia tecnológica se mide a través de nueve indicadores en el marco de tres dimensiones. Los resultados de esta investigación, contribuyen a identificar en mayor medida, la conducta y correspondencia de las variables que inciden en la transferencia tecnológica, en la mediana empresa de Cúcuta, Colombia; proporcionando información relevante, para la toma de decisiones, que permitan incrementar la competitividad de este sector.

\section{METODOLOGÍA}

La investigación realizada es descriptiva, no experimental, transaccional y de campo, puesto que permite conocer la conducta y correspondencia de las variables que inciden en la transferencia tecnológica. En este contexto según (Palella y Martins, 2012) la investigación de tipo descriptiva descifra hechos y realidades; detallando, registrando, analizando y descifrando las características y los factores en el objeto de estudio. En resumen el tipo descriptivo se enfoca en determinar los factores del momento actual. Para el presente estudio se pretende diagnosticar el hecho vigente en la mediana empresa de la ciudad de Cúcuta, Colombia.

Para el presente estudio se requiere que los datos recopilados representen las respuestas de los directivos de las empresas a una serie de preguntas (ítems) que se encuentran organizadas por categorías, con las que se pretende obtener información numérica sobre el nivel de absorción de la transferencia tecnológica en la empresa. Debido a que la investigación presenta un diseño transversal, en el cual la información recolectada sólo es válida en el periodo que se obtiene; porque ésta puede variar con el tiempo y, que además, es una investigación de campo, considerando la aplicación de un instrumento al objeto de estudio (Arias, 2006). Para tal efecto, se diseñó un instrumento estructurado con 27 criterios organizados en nueve (9) indicadores clave, los cuales son; Ciclo de vida de la tecnología, Mercado de la tecnología, Fuentes para identificar tecnología, Evaluación tecnológica, Modalidades de transferencia, Negociación y contrato de transferencia, Elementos a asimilar, Obstáculos de asimilación, y Plan de asimilación. Estos indicadores, se organizaron en un instrumento estructurado, el cual fue sometido a validación en dos fases, en la primera fase, se validó su contenido, siendo sometido a juicio de cinco (5) expertos, quienes evaluaron, la pertinencia de los ítems, con el objetivo de la investigación, su relación con la variable, la dimensión, el indicador, la coherencia, redacción, claridad conceptual y, su escala y codificación, a partir de dichas valoraciones se practicó una prueba no paramétrica siendo la prueba binomial, la que se ajustaba, se aplicó a cada una de las tres dimensiones, como se puede observar en la Tabla 1 y Tabla 2., donde se tiene que, los resultados para la Dimensión 1 (Identificación tecnológica), Dimensión 2 (Adquisición tecnológica) y, Dimensión 3 (Asimilación tecnológica), fueron similares, siendo así, se tiene que:

Grupo 1: El experto si acepta la validez de la pregunta del instrumento para la dimensión 1.

Grupo 2: El experto no acepta la validez de la pregunta del instrumento para la dimensión 1.

\section{Hipótesis estadística.}

$\mathrm{H}_{0}$ : La proporción de preguntas aceptadas en el instrumento es igual a $80 \%$

$\mathrm{H}_{1}$ : La proporción de preguntas aceptadas en el instrumento es diferente del $80 \%$

\section{Criterio de decisión.}

Se rechaza la $\mathrm{H}_{0}$ sí $\mathrm{Sig}<0.05$, caso contrario aceptar $\mathrm{H}_{0}$, luego, dado que, $\mathrm{Sig}=0.436>0.05 \mathrm{y}$ Sig $=0.134>0.05$, entonces se acepta $\mathrm{H}_{0}$.

Conclusión para prueba binomial Dimensión 1, 2 y 3. La proporción de preguntas aceptadas por los 
expertos para el instrumento de la dimensión 1, 2 y dimensión 3, es igual al $80 \%$.

Tabla 1. Prueba binomial Dimensión 1 y 3

\begin{tabular}{ccccccc}
\hline & Categoría & $\mathrm{N}$ & $\begin{array}{c}\text { Proporción } \\
\text { observada }\end{array}$ & $\begin{array}{c}\text { Proporción de } \\
\text { prueba }\end{array}$ & $\begin{array}{c}\text { Significación exacta } \\
\text { (unilateral) }\end{array}$ \\
\hline Validación de & Grupo 1 & $\mathrm{Si}$ & 8 & 0.900 & 0.800 & 0.436 \\
Experto 1 & Grupo 2 & $\mathrm{No}$ & 1 & 0.100 & 0.200 & 0.134 \\
\hline Validación de & Grupo 1 & $\mathrm{Si}$ & 9 & 1.000 & 0.800 & 0.200 \\
Experto 2 & Grupo 2 & $\mathrm{No}$ & 0 & 0.000 & 0.800 & 0.134 \\
\hline Validación de & Grupo 1 & $\mathrm{Si}$ & 9 & 1.000 & 0.200 & 0.436 \\
Experto 3 & Grupo 2 & $\mathrm{No}$ & 0 & 0.000 & 0.800 & 0.134 \\
\hline Validación de & Grupo 1 & $\mathrm{Si}$ & 8 & 0.900 & 0.200 & \\
Experto 4 & Grupo 2 & $\mathrm{No}$ & 1 & 0.100 & 0.200 & \\
\hline Validación de & Grupo 1 & $\mathrm{Si}$ & 9 & 1.000 & 0.000 & \\
Experto 5 & Grupo 2 & $\mathrm{No}$ & 0 & & 0.00 & \\
\hline
\end{tabular}

Tabla 2. Prueba binomial Dimensión 2

Categoría N Proporción observada Proporción de prueba $\begin{gathered}\text { Significación exacta } \\ \text { (unilateral) }\end{gathered}$

\begin{tabular}{|c|c|c|c|c|c|c|}
\hline \multirow{2}{*}{$\begin{array}{l}\text { Validación de } \\
\text { Experto } 1\end{array}$} & Grupo 1 & $\mathrm{Si}$ & 9 & 1.000 & 0.800 & 0.134 \\
\hline & Grupo 2 & No & 0 & 0.000 & 0.200 & \\
\hline \multirow{2}{*}{$\begin{array}{l}\text { Validación de } \\
\text { Experto } 2\end{array}$} & Grupo 1 & $\mathrm{Si}$ & 8 & 0.900 & 0.800 & 0.436 \\
\hline & Grupo 2 & No & 1 & 0.100 & 0.200 & \\
\hline \multirow{2}{*}{$\begin{array}{c}\text { Validación de } \\
\text { Experto } 3\end{array}$} & Grupo 1 & $\mathrm{Si}$ & 9 & 1.000 & 0.800 & 0.134 \\
\hline & Grupo 2 & No & 0 & 0.000 & 0.200 & \\
\hline \multirow{2}{*}{$\begin{array}{l}\text { Validación de } \\
\text { Experto } 4\end{array}$} & Grupo 1 & $\mathrm{Si}$ & 8 & 0.900 & 0.800 & 0.436 \\
\hline & Grupo 2 & No & 1 & 0.100 & 0.200 & \\
\hline \multirow{2}{*}{$\begin{array}{l}\text { Validación de } \\
\text { Experto } 5\end{array}$} & Grupo 1 & $\mathrm{Si}$ & 8 & 0.900 & 0.800 & 0.436 \\
\hline & Grupo 2 & No & 1 & 0.100 & 0.200 & \\
\hline
\end{tabular}

La segunda fase, consistió en la realización de una prueba piloto, a empresas objeto del estudio a través de un muestreo aleatorio, con la finalidad de determinar la confiabilidad del instrumento. Dicho esto, se midió la confiabilidad asociada a los 27 ítems o preguntas, calculando el coeficiente de correlación $r$ de Pearson, o también conocido como índice de discriminación (ver Tabla 3) para todos los ítems del instrumento, por otra parte, para determinar la homogeneidad de las preguntas a nivel general del instrumento, se calculó el Coeficiente Alfa de Cronbach, cuyo valor es considerado "muy bueno" con un alfa de 0.923 (Ver Tabla 3), según la escala sugerida por (Palella y Martins, 2006) quienes interpretan los resultados del coeficiente de la siguiente manera: rango 0.81 a 1.0 muy alta; rango de 0.61 a 0.80 alta; rango de 0.41 a 0.60 media; rango de 0.21 a 0.40 baja, rango de 0 a 0.20 muy baja. Según (Corral, 2009) la validez y confiabilidad hacen referencia a la fidelidad con que la población responde a las preguntas formuladas, por tanto, como se puede observar en la Tabla 3., los valores del Coeficiente $r$ de Pearson, para el total de los ítems, oscila entre 0.419 y 0.695. Según (Hernández, Fernández y Baptista, 2015) un ítem o pregunta tiene una buena relación o discrimina muy bien sí su índice de discriminación ( $r$ de Pearson) es al menos 0.40 . Esto es que, sí se encuentran entre los rangos de 0.30 y 0.39 discrimina bien, mientras que entre 0.10 y 0.29 se debería revisar. Valores inferiores cuestionan la capacidad discriminatoria del ítem. El valor promedio del índice de discriminación del instrumento es de 0.525 lo que denota un alto poder de discriminación en los ítems a nivel general. Como se puede evidenciar en la Tabla 3., los ítems i3, i6, i9, i12, i15, i18, i21, i24, i27 son los que presentan el valor de un índice discriminatorio mínimo de 0.419, sin embargo, según la escala que presentan (Hernández, Fernández y Baptista 2015) estos ítems presentan un poder discriminatorio alto. Por otra parte, analizando el Alfa de Cronbach, el efecto de excluir algún ítem del instrumento, no hará que su valor aumente, por tanto, y de acuerdo al poder de 
discriminación de los 27 ítems, los autores del presente trabajo de investigación concluyen que todos los ítems deben participar en la prueba.

Tabla 3. Análisis de confiabilidad y coeficiente Alfa de Cronbach

\begin{tabular}{|c|c|c|c|c|}
\hline ítem & $\begin{array}{l}\text { Media de } \\
\text { escala si el } \\
\text { elemento se } \\
\text { ha } \\
\text { suprimido }\end{array}$ & \begin{tabular}{lr}
\multicolumn{2}{l}{ Varianza } \\
de & escala \\
si $\quad$ el \\
elemento \\
se $\quad$ ha \\
suprimido \\
\end{tabular} & $\begin{array}{l}\text { Correlación } \\
\text { total de } \\
\text { elementos } \\
\text { corregida }\end{array}$ & $\begin{array}{l}\text { Alfa de } \\
\text { Cronbach } \\
\text { si el } \\
\text { elemento } \\
\text { se ha } \\
\text { suprimido }\end{array}$ \\
\hline i1 & 113.067 & 131.857 & 0.695 & 0.918 \\
\hline i2 & 112.633 & 137.757 & 0.492 & 0.921 \\
\hline i3 & 112.467 & 141.913 & 0.419 & 0.922 \\
\hline i4 & 113.067 & 131.857 & 0.695 & 0.918 \\
\hline i5 & 112.667 & 139.057 & 0.452 & 0.922 \\
\hline i6 & 112.467 & 141.913 & 0.419 & 0.922 \\
\hline i7 & 113.067 & 131.857 & 0.695 & 0.918 \\
\hline i8 & 112.667 & 139.057 & 0.452 & 0.922 \\
\hline i9 & 112.467 & 141.913 & 0.419 & 0.922 \\
\hline i10 & 113.067 & 131.857 & 0.695 & 0.918 \\
\hline i11 & 112.667 & 139.057 & 0.452 & 0.922 \\
\hline i12 & 112.467 & 141.913 & 0.419 & 0.922 \\
\hline i13 & 113.067 & 131.857 & 0.695 & 0.918 \\
\hline i14 & 112.667 & 139.057 & 0.452 & 0.922 \\
\hline i15 & 112.467 & 141.913 & 0.419 & 0.922 \\
\hline i16 & 113.067 & 131.857 & 0.695 & 0.918 \\
\hline i17 & 112.633 & 137.757 & 0.492 & 0.921 \\
\hline i18 & 112.467 & 141.913 & 0.419 & 0.922 \\
\hline i19 & 113.067 & 131.857 & 0.695 & 0.918 \\
\hline i20 & 112.667 & 139.057 & 0.452 & 0.922 \\
\hline i21 & 112.467 & 141.913 & 0.419 & 0.922 \\
\hline i 22 & 113.067 & 131.857 & 0.695 & 0.918 \\
\hline i23 & 112.667 & 139.057 & 0.452 & 0.922 \\
\hline i24 & 112.467 & 141.913 & 0.419 & 0.922 \\
\hline i25 & 113.067 & 131.857 & 0.695 & 0.918 \\
\hline i26 & 112.667 & 139.057 & 0.452 & 0.922 \\
\hline i27 & 112.467 & 141.913 & 0.419 & 0.922 \\
\hline \multicolumn{2}{|c|}{ Alfa de Cronbach } & 0.923 & & \\
\hline \multicolumn{2}{|c|}{$\mathrm{N}^{\circ}$ de elementos } & 27 & & \\
\hline
\end{tabular}

Para determinar la población, se clasificaron las empresas según los activos totales, filtrando de la base de datos de la Superintendencia de Sociedades de Colombia, para el departamento Norte de Santander y acotando geográficamente la zona para la ciudad de Cúcuta, teniendo en cuenta las clasificaciones del Código Industrial Internacional Uniforme (CIIU). De acuerdo a dicho criterio, se obtuvo una población finita de 214 empresas de tamaño mediana.

Buscando que los resultados obtenidos sean representativos para la población, se calculó la muestra de estudio, por lo que se empleó un porcentaje de error del $5 \%$, un nivel de confianza del $95 \%$, y un nivel de probabilidad de éxito y fracaso del $50 \%$ para cada uno, respectivamente. Aplicando la Ecuación (1)

$$
n=\frac{p * q * z^{2} * N}{N * \varepsilon^{2} * Z^{2} * p * q}
$$

Done $n$ es el número de elementos de la muestra, $N$ es el número de elementos de la población, $p$ y $q$, son respectivamente el éxito y el fracaso asociado al evento, $Z$ es el valor crítico correspondiente al nivel de confianza suponiendo una distribución normal y finalmente $\varepsilon$ es el margen de error permitido.

Para la obtención de la información del instrumento, inicialmente, se contactó con los directivos de las empresas por medio de correo electrónico, para sensibilizar al personal objeto de estudio, a través de herramientas pedagógicas sobre la importancia del estudio de investigación, seguidamente se aplicó el instrumento vía E-mail o en formato encuesta directa (visita en la empresa). Se indicó a los responsables de responder el instrumento, leer cuidadosa y detenidamente cada una de las afirmaciones, con la finalidad de conocer su nivel de apreciación respecto a la pregunta del ítem. En el estudio se aplica un cuestionario estructurado, veintisiete (27) preguntas cerradas, con una escala Likert, que pretende medir el nivel de apreciación del encuestado, con respecto al cumplimiento de las afirmaciones que se plantean de la siguiente manera: Nunca (1), Casi nunca (2), A veces (3), Casi siempre (4), y Siempre (5). Obteniéndose así, información de 138 empresas. El análisis de la información se efectuó mediante estadística descriptiva, tabulando los datos en la herramienta Microsoft Excel ${ }^{\circledR}$, y con el software SPSS del que se obtuvo los reportes estadísticos.

\section{RESULTADOS}

Los resultados son confrontados con los referentes teóricos que sustentan la investigación, con el fin de diagnosticar las variables, dimensiones e indicadores estudiados. 


\subsection{Variable: Transferencia tecnológica}

La transferencia de tecnología, está definida generalmente como una herramienta, y su discusión está orientada a establecer qué tipo de herramienta puede ser considera como tecnología (Bozeman, 2000).

La transferencia tecnológica germina como una herramienta para marcar diferencias notorias entre las organizaciones, como ventaja competitiva, con el propósito de identificar las características de las empresas y conocer qué tipo de tecnologías del contexto son apropiadas para su mejora, y qué tipo de tecnologías puede la organización contribuir favorablemente al entorno (Domínguez, 2012).

Según (Leal, 2012), la transferencia tecnológica se define como los mecanismos y procedimientos de adquisición de conocimientos tecnocientíficos, como herramienta para implantar un desarrollo industrializado, a través de la fabricación de productos y diseño de servicios; requeridos de acuerdo con las necesidades y problemáticas autóctonas.

Considerando las interpretaciones expuestas, es posible entender que la transferencia tecnológica es un proceso con la capacidad de adaptarse a las necesidades del contexto, y existe cuando hay apropiación del conocimiento y éste es aceptado por el mercado.

De acuerdo con la revisión bibliográfica, (Gaynor, 1996), (Slowinski, 2000), (Phillips, 2001), (Steenhuis y Boer, 2002), (Cepal, 2013), (Conpes, 2015) y (Unasur, 2014) se puede representar a la transferencia tecnológica en una empresa mediante un proceso continuo que incorpora tres dimensiones denominadas: identificación, adquisición y asimilación, como se muestra en la Figura 1.

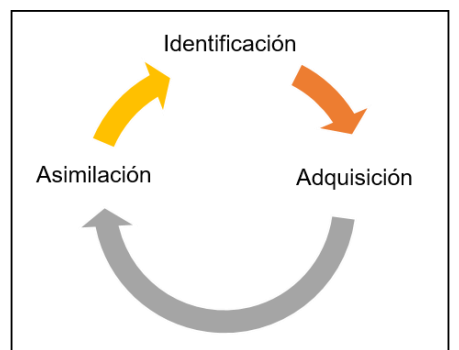

Fig. 1. Modelo de transferencia tecnológica.

Para el desarrollo de la presente investigación y brindar una interpretación a los resultados obtenidos, se elaboró un baremo para la interpretación de las variables de estudio como se aprecia en la Tabla 4., el cual relaciona el grupo, el rango de los valores y el atributo requeridos para el análisis del promedio, considerando las cinco (5) posibilidades.

Tabla 4. Baremo para interpretar la media

\begin{tabular}{ccc}
\hline Grupo & Rango de valores & Atributo \\
\hline 5 & $4.2 \leq \mathrm{R} \leq 5.0$ & Muy bueno \\
4 & $3.4 \leq \mathrm{R} \leq 42$ & Bueno \\
3 & $2.6 \leq \mathrm{R} \leq 3.4$ & Regular \\
2 & $1.8 \leq \mathrm{R} \leq 2.6$ & Deficiente \\
1 & $1.0 \leq \mathrm{R} \leq 1.8$ & Muy deficiente \\
\hline
\end{tabular}

\subsubsection{Dimensión: Identificación tecnológica -} Indicador: Ciclo de vida de la tecnología.

Para evaluar el indicador ciclo de vida de la tecnología el instrumento plantea tres ítems o preguntas, analizando las respuestas cuantitativamente de acuerdo con el baremo para la interpretación de la media (ver Tabla 4), el indicador ciclo de vida de la tecnología es homogéneamente regular con media igual a 3.23 (ver Tabla 5). Las respuestas evidencian que la mediana empresa de Cúcuta aplica aceptablemente el ciclo de vida de la tecnología; es decir, de acuerdo con (Benavides, 1998) no asimila adecuadamente el proceso evolutivo de la tecnología, lo que redunda en un desperdicio de su rendimiento y vida útil.

\subsubsection{Dimensión: Identificación tecnológica - Indicador: Mercado de la tecnología.}

En el análisis estadístico del indicador mercado de la tecnología, se evidenció que es un indicador regular, según el baremo para el análisis de las medias y la varianza; con media del indicador igual a 3.50 y varianza de 0.179 . Las respuestas manifiestan que la mediana empresa de Cúcuta no define con claridad la motivación de la transferencia de la tecnología y la orientación de los esfuerzos físicos, financieros y de tiempo (Benavides, 1998).

\subsubsection{Dimensión: Identificación tecnológica - Indicador: Fuentes para identificar tecnología.}

Este indicador presenta una media calificada como regular y una varianza de 0.269 producto de una respuesta calificada como buena y dos como regular (ver Tabla 5). Las respuestas permiten inferir que gran porcentaje de las medianas empresas de Cúcuta utilizan fuentes de 
información de libre disposición para identificar tecnología, la cual normalmente está asociada con tecnologías maduras u obsoletas (Tapias, 1996). Además, se alinean regularmente las fuentes de información no libre, en donde se destacan las tecnologías emergentes y claves, con la participación en redes de negocio y de investigación (CONPES, 2015).

\subsubsection{Dimensión: Adquisición tecnológica. - Indicador: Evaluación tecnológica.}

El indicador evaluación tecnológica está valorado como regular con media de medias de 3.74 y varianza de 0.073 (ver Tabla 5), dos respuestas son valoradas con atributo bueno y una respuesta con regular. Es posible evidenciar en las respuestas que una buena representación de las empresas en estudio realiza una evaluación de la factibilidad técnica de transferir la tecnología; sin embargo, se le da menor importancia a la evaluación del mercado, lo cual reduce la posibilidad de adaptación de la tecnología. Se identifica que la mediana empresa de Cúcuta busca tecnologías con futuro crecimiento potencial, con margen de utilidad superior al promedio y un rápido retorno de la inversión.

\subsubsection{Dimensión: Adquisición tecnológica. - Indicador: Modalidades de transferencia.}

El indicador modalidades de transferencia, fue calificado con 3.69 y valorado como bueno. Las tres preguntas planteadas por este indicador tienen respuestas con media valorada en bueno. Con base en las repuestas y según (Rodríguez, 2014) es posible afirmar que las medianas empresas de Cúcuta aceptan de buena manera la compra de productos que incorporan la tecnología en dicho producto. De acuerdo con (CEPAL, 2013) el resultado para este indicador refiere a que las empresas objeto de estudio, utilizan en buen porcentaje las alianzas con otras compañías como estrategia para acceder a tecnologías y mercados, con menor riesgo y costo. Se observa que las organizaciones encuestadas utilizan en buena medida el licenciamiento para adquirir el conocimiento y los derechos de la tecnología, aunque éstos se encuentres limitados a un periodo de tiempo y sector del mercado.

3.1.6 Dimensión: Adquisición tecnológica. -
Indicador: Negociación y contrato de
transferencia.
transferencia.
El indicador es valorado en general como bueno con media de 3.96 y varianza 0.53 (ver Tabla 5); lo que indica que las respuestas reflejan que al adquirir tecnología las empresas en su mayoría no contratan personal externo para apoyar el proceso de negociación; contemplan en la negociación del precio de la tecnología las ventas, las ganancias y la realidad del mercado, entre otros; y resguardan los acuerdos pactados mediante contratos e instrumentos. Estas consideraciones promueven que la transferencia tecnológica se desarrolle de manera acorde.

\subsubsection{Dimensión: Asimilación tecnológica. - Indicador: Elementos a asimilar.}

Analizando las respuestas cuantitativamente de acuerdo con el baremo para la interpretación de la media, el indicador elementos a asimilar es homogéneamente regular con media 3.90 y varianza de 0.001 , del análisis estadístico se puede deducir que la mediana empresa de Cúcuta tiene una dependencia u oficina de tecnología o que le asignó estas funciones a alguna dependencia; que se preocupa en el proceso de asimilación por los elementos tangibles e intangibles; y direcciona esfuerzos humanos, de infraestructura, económicos y de mercado, para contribuir a la adaptación de la nueva tecnología.

\subsubsection{Dimensión: Asimilación tecnológica. - Indicador: Obstáculos de asimilación.}

Este indicador es disperso en sus resultados, tiene una media de 3.66 , con una varianza de 0.406 y está valorado como bueno, esto es que la mediana empresa de Cúcuta con respecto a la asimilación tecnológica reconoce que implica cambios relevantes en la cultura organizacional; y que se ve truncada por el grado de educación de los empleados y por la sostenibilidad ecológica del entorno en donde ésta se aplica

\subsubsection{Dimensión: Asimilación tecnológica. - Indicador: Plan de asimilación.}

En este indicador todas las respuestas son valoradas como bueno y su media es de 3.77 con atributo bueno y una varianza de 0.004 (ver Tabla 5). Es posible inferir que las empresas objeto de estudio de la ciudad de Cúcuta, dan importancia a la elaboración del plan de asimilación con el propósito de mitigar o evitar los obstáculos relevantes del proceso de transferencia de la tecnología, asignando personal responsable de múltiples disciplinas en las compañías receptora y 
cedente que contribuyan a la apropiación de los distintos aspectos de la transferencia tecnológica.

Tabla 5. Diagnóstico global de la variable transferencia tecnológica

\begin{tabular}{|c|c|c|c|c|c|c|c|c|c|c|}
\hline Indicador & Media & Atributo & Dimensión & Media & Atributo & $\sigma^{2}$ & Variable & Media & Atributo & $\sigma^{2}$ \\
\hline $\begin{array}{l}\text { Ciclo de vida de la } \\
\text { tecnología }\end{array}$ & 3.23 & Regular & \multirow{3}{*}{$\begin{array}{l}\text { Identificación } \\
\text { tecnológica }\end{array}$} & \multirow{3}{*}{3.38} & \multirow{3}{*}{ Regular } & \multirow{3}{*}{0.013} & & \multirow{9}{*}{3.65} & \multirow{9}{*}{ Bueno } & \multirow{9}{*}{0.037} \\
\hline $\begin{array}{l}\text { Mercado de la } \\
\text { tecnología }\end{array}$ & 3.50 & Bueno & & & & & & & & \\
\hline $\begin{array}{l}\text { Fuentes para } \\
\text { identificar } \\
\text { tecnologías }\end{array}$ & 3.39 & Regular & & & & & & & & \\
\hline $\begin{array}{l}\text { Evaluación } \\
\text { tecnológica }\end{array}$ & 3.74 & Regular & & & & & & & & \\
\hline $\begin{array}{l}\text { Modalidades } \\
\text { transferencia }\end{array}$ & 3.69 & Bueno & $\begin{array}{l}\text { Adquisición } \\
\text { tecnológica }\end{array}$ & 3.80 & Bueno & 0.014 & $\begin{array}{l}\text { Transferencia } \\
\text { Tecnológica }\end{array}$ & & & \\
\hline $\begin{array}{l}\text { Negociación } \\
\text { contrato } \\
\text { transferencia }\end{array}$ & 3.96 & Bueno & & & & & & & & \\
\hline Elementos a asimilar & 3.90 & Bueno & \multirow{3}{*}{$\begin{array}{l}\text { Asimilación } \\
\text { tecnológica }\end{array}$} & \multirow{3}{*}{3.78} & \multirow{3}{*}{ Bueno } & \multirow{3}{*}{0.009} & & & & \\
\hline $\begin{array}{l}\text { Obstáculos } \\
\text { asimilación }\end{array}$ & 3.66 & Bueno & & & & & & & & \\
\hline Plan de asimilación & 3.77 & Bueno & & & & & & & & \\
\hline
\end{tabular}

\section{CONCLUSIONES}

La mediana empresa de Cúcuta, Colombia, en la transferencia tecnológica, identifica la tecnología de manera regular, lo que implica una concepción regular del ciclo de vida de la tecnología y de las fuentes para identificar la tecnología, y una concepción buena del mercado de la tecnología. Es decir, la mediana empresa de Cúcuta aceptablemente: (i) utiliza tecnología de punta, la cual es posible adquirir en el mercado internacional desde hace menos de 5 años; (ii) utiliza tecnología la cual no es más avanzada con respecto a la de la competencia nacional; (iii) tiene actividad organizada para identificar tecnologías claves que beneficien a la compañía; (iv) prioriza que los productos tecnológicos a adquirir tengan una posible adaptación para condiciones de uso diferentes; (v) compra tecnologías en mercados nacionales; (vi) identifica tecnologías a través de fuentes de información no libres cuya propiedad está legalmente protegida; y (vii) su personal participa en redes de negocio o de investigación. De igual forma, la mediana empresa de Cúcuta de manera buena: (i) define con claridad la motivación de la compañía para adquirir tecnología y (ii) con frecuencia identifica la tecnología a través de fuentes de información libre como libros, revistas, patentes de invención vencidas, entre otras.
La mediana empresa de Cúcuta, en la transferencia tecnológica, en términos generales cumple de buena manera con el proceso de adquisición de la tecnología. Sin embargo desagregando el concepto de adquisición tecnológica, es posible decir que regularmente: (i) la evaluación del mercado incluye el tamaño del producto, las tendencias, la madurez, el crecimiento, la estructura competitiva, las características socio culturales, los clientes que sustentan la tecnología, entre otros; y (ii) no requiere de personal externo para apoyar la negociación y el trato. De igual manera se puede inferir que la mediana empresa de Cúcuta al adquirir tecnología, de buena manera: (i) evalúa con rigurosidad técnica la tecnología para que cumpla con los requerimientos y se garantice su integración en los procesos; (ii) evalúa económicamente un retorno de inversión en un tiempo no mayor a los tres años; (iii) adquiere la tecnología mediante la compra de un producto de una empresa que incorpora la tecnología en dicho producto y que, por tal motivo, se transmite con él; (iv) establece alianzas con otras empresas para acceder a tecnologías y a mercados; y (v) recurre al licenciamiento como modalidad de transferencia para adquirir el conocimiento y los derechos de una tecnología. Finalmente la mediana empresa en estudio, cuando adquiere tecnología, de muy buena manera: (i) considera en la negociación del precio 
de la tecnología la realidad del mercado, las ventas, las ganancias, entre otros; y (ii) utiliza contratos o instrumentos que protegen los acuerdos negociados (contrato de compraventa, de licenciamiento o exclusividad de la tecnología)

En la fase de asimilación de la transferencia tecnológica la mediana empresa de Cúcuta reconoce los elementos a asimilar, los obstáculos presentes en la asimilación y la exigencia de elaborar un plan de asimilación. Al respecto, el estudio permite desagregar que en buenos términos la mediana empresa investigada: (i) asimila la tecnología haciendo uso de un departamento tecnológico; (ii) considera la asimilación de elementos intangibles, como la información tecnológica, científica y de desarrollo no agregada directamente en los equipos tangibles; (iii) emplea los recursos necesarios (humanos, administrativos, infraestructura, económicos y mercado) para garantizar las adaptaciones exigidas por la nueva tecnología; (iv) realiza cambios significativos en la cultura organizacional; (v) contempla un plan de asimilación en los acuerdos de la transferencia tecnológica; (vi) elabora un plan de asimilación de la tecnología con personas responsables en las empresas receptora y cedente; y (vii) reúne personas de múltiples profesiones, que facilitan la apropiación de los diferentes aspectos de la transferencia. Además, en la mediana empresa de Cúcuta la asimilación de la tecnología se ve obstruida por el nivel de educación de los empleados y por la sostenibilidad ecológica del contexto en donde ésta se aplica.

\section{REFERENCIAS}

CEPAL, (2011) Comisión Económica para América Latina y el Caribe, Políticas para la innovación en las pequeñas y medianas empresas en América Latina. Santiago de Chile: CEPAL.

F. J. Vélez V (2006) Estudio sobre la informalidad $y$ las MIPYMES en Colombia: Análisis y propuestas, Bogotá: OIT.

Rodríguez, L. G. (2014) Lecciones aprendidas sobre adopción de TIC en PYMES: la experiencia del FOMIN/BID en América Latina, Fundación Telefónica Venezuela, pp. 11-12, República Bolivariana de Venezuela.

OCDE/CEPAL (2012), Perspectivas Económicas de América Latina 2012: Transformación del Estado para el Desarrollo, OECD Publishing. http://dx.doi.org/10.1787/leo-2012.
Del Río Cortina, A., Salamanca, Y. D. T., Amortegui, B. X. A., \& Martínez, K. N. R. (2016). "Estudio de variables de gestión del talento humano basada en competencias". Mundo FESC No 11.

CEPAL, Comisión Económica para América Latina y el Caribe (2013) Cómo mejorar la Competitividad de las pymes en la Unión Europea y América Latina y el Caribe. Santiago de Chile: CEPAL.

Jaimes, M., Ramírez D., Vargas, A., y Carrillo, G. (2011). "Estado del arte vigilancia tecnológica: una aplicación para la innovación". Revista Gerencia Tecnológica Informática Vol. 10, No. 26.

Colciencias, Instituto Colombiano para el Desarrollo de la Ciencia y la Tecnología "Francisco José de Caldas" (2005) Plan estratégico del programa nacional de desarrollo tecnológico, industrial y calidad 2005 - 2015. Bogotá: Colciencias.

León, J. A. P., Osorio, L. M. B., \& Andrade, J. M. M. (2015). "Valor económico agregado en las empresas del sector industrial manufacturero de Cúcuta periodo 2008-2012”. Respuestas, Vol. 20 No 2.

Roncancio-Lozano, M. A., Castro-Martin, J. A., \& Rivera-Basto, A. (2015). "Análisis comparativo de las normas ISO 9001:2008, ISO 14001:2004 y OHSAS 18001:2007, para su aplicación integral en procesos de construcción para empresas de Ingeniería Civil". Respuestas Vol. 20 No 1.

CONPES, Consejo Nacional de Política Económica y Social (2015) Política Nacional de Ciencia, Tecnología e Innovación, 20152025, República de Colombia.

Morales-Castro, J. A. (2015). "La acreditación de sustentabilidad y su impacto en los márgenes de rentabilidad del sector industrial de la Bolsa Mexicana de Valores". Respuestas, Vol. 20 No 1.

Becerra, E. S. (2017). "Análisis de los potenciales efectos en el reconocimiento de los activos de las pequeñas empresas de Cúcuta-Colombia por la implementación de la NIIF para Pymes". Respuestas, Vol. 22 No 2.

Kim, Linsu. (1999). Learning and innovation in economic development. Edward Elgar Publishing, Inc., Northampton Massachusetts, USA.

Ulrich, K. T. (2000). Product Design and Development. 2nd Ed. McGraw-Hill. International Edition. 
Rojas, P. R. S. (2016). "Impacto socioeconómico a un año del cierre de la frontera ColomboVenezolana: Norte de Santander-Estado Táchira (2015-2016)". Mundo FESC Vol. 6 No 12.

Rodríguez, L. J., \& Gamboa-Suárez, R. (2018). “El capital humano e intelectual como catalizador de la gestión organizacional". Mundo Fesc Vol 8 No 15.

Moreno, J. R. V., Rincón, G. M. L., \& Linares, Á. S. (2016). "La importancia de formalizar empresa con potenciales emprendedores de la localidad de Bosa, bajo un entorno de globalización económica y desarrollo social”. Mundo Fesc No 11.

Palella, S y Martins, F. (2012). Metodología de la investigación cuantitativa 3ra Ed (2012). FEDEUPEL. Caracas. Venezuela.

Arias, F. (2006). El Proyecto de Investigación. Introducción a la Metodología científica. Quinta edición. Caracas: Editorial Episteme.

Corral, Y. (2009). "Validez y confiabilidad de los instrumentos de investigación para la recolección de datos". Revista Ciencias de la Educación. Vol. 19. № 33.

Hernández, R., Fernández, C. y Baptista, M., (2010) Metodología de la Investigación, Quinta edición, McGraw-Hill/Interamericana Editores, México.

Bozeman, B. (2000). "Technology transfer and public policy: a review of research and theory". Research Policy. Vol 29 No 4-5.

Domínguez, Karen. (2012) Desarrollo de un modelo de transferencia tecnológica para empresas de base tecnológica. Caso de estudio: Corporación de ciencia y tecnología para el desarrollo de la industria naval, marítima y fluvial - COTECMAR. Universidad Tecnológica de Bolívar, Cartagena de Indias.

Leal, Víctor. (2012) Transferencia internacional de tecnología para el desarrollo endógeno en el sistema energético de Venezuela. Universidad Dr. Rafael Belloso Chacín, Maracaibo, Vzla.

Gaynor, Gerard (1996). Handbook of technology management. New York: McGraw-Hill.

Slowinski, Gene. (2000). "Acquiring external technology. Research" Technology Management. Washington. Vol. 43. No 5.

Phillips, Fred. Y. (2001). Market-oriented technology management: innovating for profit in entrepreneurial times. New York: Springer.
Steenhuis, H. J. \& de Boer, S. J. (2002). "Differentiating between types of technology transfer: the technology building". International Journal of Technology Transfer and Commercialization Vol 1, No 1-2.

Benavides, Carlos (1998). Tecnología, innovación y empresa. Madrid: Editorial Pirámide.

Tapias, Heberto. (1996). "Transferencia de tecnología”. Revista Facultad de Ingeniería, ISSN 0120-6230 Vol 3 No. 12. 